\title{
La terapia nella malattia di Fabry
}

\author{
Renzo Mignani
}

\section{Editoriale}

Con questo numero del Giornale delle Tecniche Nefrologiche e Dialitiche inizia la pubblicazione di una serie di articoli riguardanti la terapia specifica per la malattia di Fabry. Come per ogni patologia, ma in modo particolare per le malattie rare, la terapia rappresenta un aspetto fondamentale per i pazienti che ne sono affetti e per tale motivo il comitato editoriale di tale rubrica ha pensato di darle ampio spazio dedicando un articolo alla terapia enzimatica, uno alla terapia chaperonica e un terzo alle nuove terapie non ancora in commercio ma che si stanno affacciando all'orizzonte.

La terapia enzimatica sostitutiva con agalsidasi è stato il primo trattamento introdotto per la malattia e, a distanza di quasi vent'anni, è ampiamente dimostrato come tale farmaco sia in grado di prevenire $o$, al limite, di stabilizzare la malattia arrestandone la progressione e modificando cosi la storia naturale della malattia. Il trattamento con l'agalsidasi determina infatti una significativa riduzione dell'accumulo di $\mathrm{Gb} 3$, migliora la prognosi cardiaca e stabilizza la funzione renale. Ne risulta un significativo miglioramento della qualità della vita sia nei maschi ma anche nelle femmine e in modo particolare nei bambini e negli adolescenti per la notevole efficacia di tale farmaco sul dolore neuropatico. Ma è altrettanto dimostrato che l'efficacia di tale terapia dipenda principalmente dalla tempestività con cui viene introdotta, poiché più precocemente viene avviata maggiore è la possibilità di rimuovere l'accumulo cellulare prima che ciò abbia determinato il danno tissutale e la conseguente insufficienza funzionale dell'organo. Inoltre la terapia enzimatica ha dimostrato la capacità di ridurre in modo significativol'incidenza degli eventicardiaci e cerebrovascolari migliorando sensibilmente la morbidità di tale malattia. Tuttavia rimangono aperti alcuni problemi riguardanti tale terapia come la necessità della somministrazione endovenosa, l'obbligo del trattamento ospedaliero dove non è possibile eseguirla al proprio domicilio, la possibile formazione di anticorpi neutralizzanti o la possibile intolleranza al farmaco, temi che verranno affrontati ed approfonditi nel primo capitolo pubblicato in questo numero del giornale.

Nel prossimo numero del giornale si parlerà invece della terapia chaperonica, la prima terapia orale introdotta per la malattia di Fabry. Tale farmaco, in commercio anche in Italia da un paio d'anni, oltre al vantaggio dell'assunzione
Giornale di Tecniche Nefrologiche e Dialitiche 2019, Vol. 31 (3) 206

(C) The Author(s) 2019

Article reuse guidelines:

sagepub.com/journals-permissions

DOI: 10.1177/0394936219869174

journals.sagepub.com/home/gtn orale, ha dimostrato negli studi registrazione una notevole efficacia soprattutto sul coinvolgimento cardiaco. Purtroppo però, ne possono beneficiare solo i pazienti con mutazioni suscettibili, quelli in cui continua ad essere prodotta una minima quantità di enzima $\alpha$-galattosidasi. Inoltre, alcune categorie di pazienti come i bambini, gli adolescenti e gli adulti affetti da una severa insufficienza renale ne sono al momento esclusi. Tuttavia, sono in corso studi più approfonditi e soprattutto prolungati allo scopo di confermare i risultati ottenuti negli studi di registrazione anche in queste popolazioni di pazienti.

Infine nel terzo numero del Giornale verranno trattati i farmaci che sono ancora in sperimentazione ma che entro 3-4 anni si ritiene possano aggiungersi all'armamentario terapeutico per la malattia di Fabry. Tra questi ricordiamo il pegunigalsidase alfa, una forma pegylata dell'agalsidasi, di origine vegetale e a somministrazione endovenosa. Il vantaggio ipotizzato per tale farmaco, rispetto alle due formulazioni di agalsidasi alfa e beta attualmente in commercio sarebbe una più lunga emivita che consentirebbe una maggiore biodisponibilità tissutale, e soprattutto una minor immunogenicità anticorpale. Un altro farmaco in corso di studio è il Lucerastat. Si tratta di un iminozucchero che attraverso l'inibizione dell'enzima glucosylceramide sintetasi riduce la produzione dei glicosfingolipidi. Il vantaggio di tale terapia, anch'essa orale, sarà che tutti $\mathrm{i}$ pazienti ne potranno beneficiare indipendentemente dal tipo di mutazione presente. Infine, sono in corso sperimentazioni con la terapia genica. Tale trattamento si basa sulla raccolta e successiva reintroduzione nel paziente di proprie cellule staminali che vengono incubate con un lentivirus che funziona da vettore essendogli stato inserito una copia sana del gene GLA. Al momento sono in corso alcune sperimentazioni su di un numero limitato di pazienti ma le aspettative sono molto elevate per il successo di una terapia che potrebbe cambiare radicalmente la storia naturale della malattia e la vita di tali pazienti.

AIAF, UO Nefrologia e Dialisi, Ospedale Infermi, Rimini, Roma, Italy

\section{Corrispondenza:}

Renzo Mignani, UO Nefrologia e Dialisi Ospedale Infermi, Rimini

Coordinatore Comitato Scientifico AIAF, Italy.

E-mail: renzo.mignani@auslromagna.it 